\title{
Difference in Outdoor Time and Physical Activity During Recess After Schoolyard Renewal for the Least-Active Children
}

\author{
Charlotte Skau Pawlowski, Henriette Bondo Andersen, and Jasper Schipperijn
}

\begin{abstract}
Background: It remains unclear if schoolyard interventions "just" provide more opportunities for those children who are already active. The authors wanted to investigate schoolyard use and physical activity (PA) among the least-active children during recess following schoolyard renewals. Methods: An intervention study design with preresults and postresults comparison was used. Accelerometer and global positioning system data were collected at 6 Danish schools from 553 children at baseline and 439 after renewals (grades 4-9). Based on mean minutes of recess moderate to vigorous PA per child per school, the least-active children were defined as all children in the lowest activity quartile at baseline and follow-up, respectively. Results: One hundred and thirty-five children (70\% girls) at baseline and 108 (76\% girls) at follow-up were categorized as the least-active children. At follow-up they accumulated more time $(12.1 \mathrm{~min} / \mathrm{d})$ and PA $(4.4 \mathrm{~min} / \mathrm{d})$ in the schoolyard during recess compared with baseline. The difference in schoolyard PA found for the least-active children was relatively small compared with the difference for all children. Conclusions: Solely improving the physical schoolyard environment seemed to have limited impact on the least-active children's PA. Future studies should investigate the complex interrelations between the least-active children and the entire schoolyard environment.
\end{abstract}

Keywords: built environment, schoolyard intervention, GPS, accelerometry

Physical activity (PA) in childhood is associated with a multitude of health benefits. ${ }^{1}$ In spite of the growing awareness of these benefits, a large number of school children do not reach the recommended minimum level of 60 minutes of moderate to vigorous PA (MVPA) per day in Denmark and other western countries. ${ }^{2}$ To prevent chronic diseases, children are encouraged to participate in PA while they are young and to continue into adulthood. ${ }^{3}$ Health professionals are focusing on the least-active children to get these children "back on track" from the beginning. ${ }^{1}$

School recess is a key setting that can provide opportunities for children to play and be active. ${ }^{4,5} \mathrm{~A}$ review revealed that recess can contribute between 5\% and $40 \%$ of children's recommended daily PA levels. ${ }^{4}$ Furthermore, studies have found the least-active children to accumulate most of their daily PA during recess. ${ }^{6-8}$ Thus, recess PA might be a valuable contribution to overall school day PA for the least-active children.

To further increase the contribution to overall school day PA, a variety of recess interventions have been reported including addition of schoolyard equipment, structured activities, teacher involvement, and playground markings (ie, designs that can be laid, sprayed, and heated into a surface like hopscotch, sports court lines, and figures with numbers and letters). ${ }^{9}$ Among these, the largest change between children's PA levels before and after intervention occurred with an addition of playground equipment. ${ }^{9}$ However, only few recess intervention studies have investigated separate groups of children based on their baseline PA levels. ${ }^{10,11}$ One intervention study redesigning the schoolyard with playground markings found that the intervention effect was stronger for children who engaged in less MVPA at baseline. ${ }^{10}$ In another study, investigating the effect of playground markings and physical

The authors are with the Research unit for Active Living, Department of Sports Science and Clinical Biomechanics, University of Southern Denmark, Odense, Denmark. Pawlowski (cspawlowski@health.sdu.dk) is corresponding author. structures, children's individual PA levels at baseline was not found to be a predictor for their recess PA after intervention. ${ }^{11}$ As only few previous schoolyard intervention studies have investigated the effect of different groups of children based on their baseline PA levels and with mixed results, it remains unclear if these structural interventions are equally effective for all children, or if they "just" provide more opportunities for those children who are already active. As far as we know, no schoolyard intervention studies have focused specifically on the least-active children's recess PA behavior.

The aim of this study was to investigate schoolyard use and PA among the least-active children during recess following schoolyard renewals. Our research questions were: (1) Will the least-active children spend more time in the schoolyard during recess after renewals; and (2) Will the least-active children accumulate more recess $\mathrm{PA}$ in the schoolyard after renewals?

\section{Method}

\section{Study Design}

This study is part of the Activating Schoolyards Study, an intervention study design with preresults and postresults comparison. The aim of the study was to evaluate the effect, process, and user perspective of schoolyard renewals. To provide a comprehensive evaluation, a range of quantitative (accelerometers, global positioning system [GPS] devices, and survey) and qualitative methods (interviews and observation) were used at baseline (April to June 2014) and follow-up (April to June 2016). ${ }^{12}$ About 10- to 16-yearold children (grades 4-9), representing the target group for the schoolyard renewal at each school, participated at the 2 time points. They were not followed overtime to avoid the age-dependent decline in PA. ${ }^{13}$ In the current study, we explicitly focused on the least-active children at the 2 time points. 


\section{The Danish Recess Context}

In Danish schools, children in grades 4 to 9 have approximately 60 to 75 minutes recess per day, being distributed over 2 to 4 breaks. In general, the lunch break is the longest break, lasting approximately 30 minutes. Recess is typically characterized by free play without any organized curriculum. Teachers on schoolyard duty are supervising the students, handling conflicts, and accidents. There is wide variation in whether schools have an outdoor recess policy for children in grades 4 to 9 , but most often this group of children is allowed to stay indoors during at least some recess periods a day.

\section{Recruitment of Schools}

The schools were selected based on an open call for proposals issued by a partnership consisting of 3 nongovernmental organizations. In October 2012, all public primary and lower secondary schools in Denmark (approximately 1800 schools) were invited to submit a vision proposal. Among 106 submitted proposals, the partnership selected 7 projects for realization in February 2014.

One project was delayed beyond the study timeline and was not included in the follow-up data collection, and therefore excluded from this paper. The 6 remaining projects were realized at schools varying in size, geographical location (urban or rural), parental socioeconomic status, ethnicity, schoolyard size, number of play facilities, number of recess periods, total recess duration, and recess rules (eg, a policy obliging children to stay outdoors parts of their recess were presented at 3 schools). ${ }^{12}$

\section{Intervention}

The schoolyard renewals were developed using a participatory bottom-up approach involving children in the development process. It was up to the schools to decide how the children participated in the process. At some schools, architects were responsible for workshops with children, and at other schools, a process consultant was hired to facilitate the involvement with children. Number, age, and characteristics of children included in the process differed among the schools, as did the time spent, and techniques used to develop with the children. No schools recruited children to take part in the development process based on their recess PA levels.

The design and dimension of the intervention components varied widely, reflecting the local challenges and needs. ${ }^{12}$ At some of the schools the changes took place in the existing schoolyard, whereas other schools expanded their outdoor area by including adjacent spaces (eg, woodland area and parking ground). The most essential change was implementation of new play features in the schoolyard such as climbing walls, balance-bars, theater/dancingstages, skating areas, trampolines, hills, and ball game facilities, that were applied at most of the schools. ${ }^{14}$ Descriptions of intervention components and photos of the 6 schoolyards after renewal are provided in Table 1 . The renewals took place during 2015, and the total budget for each of the schools ranged from 120,000 to 900,000 USD.

\section{Data Collection Procedures}

To assess the difference in schoolyard use and PA during recess before and after the schoolyard renewals, a combination of accelerometers, GPS, geographic information system (GIS), and class timetables were used. It is found applicable to use a combination of these methods to measure the relationship between PA and environment in children. ${ }^{15}$ The device-based PA data were measured as an activity count every 15 seconds using the ActiGraph (ActiGraph, Pensacola, FL) accelerometer (model GT3X) to explore differences in PA intensity levels during recess. ${ }^{16}$ The children's locations during recess were recorded every 15 seconds using QStarz BT-Q1000xt GPS trackers, which have a small median dynamic positional error of $2.9 \mathrm{~m} .{ }^{17}$ The schoolyard was mapped using GIS software (version 10.2; ArcGIS, Esri, Redlands, CA), and the total outdoor area at the school was calculated. For this paper, the GPS and GIS data were used to determine if and how long the children used the schoolyards during recess before and after the intervention.

The children were asked to wear the accelerometer and GPS in an adjustable elastic belt on their waist for 7 consecutive days (5 schooldays) during the 2 data collection periods. Verbal and written instructions on how to wear the equipment were given to the children by the research team. The equipment was not worn overnight and during water-based activities. To increase compliance, the children received short reminder text messages on their mobile phones twice a day, asking them to take the belt off over night, when the GPS was charged, and to put the belt on in the morning.

\section{Ethics}

The study was registered in The International Standard Randomized Controlled Trial Number registry (ISRCTN17944407), and its data management procedures were approved by the Danish Data Protection Agency (2014-41-2801). According to the Danish National Committee on Health Research Ethics, formal ethical approval was not required, as the project was not a biomedical research project. All parents of the participating children provided a written informed consent on behalf of their children for each wave of data collection, and all children provided their assent and could withdraw from the study at any time.

\section{Data Analysis}

The accelerometer and GPS data were downloaded using ActiLife (version 6.11.4; ActiGraph, Pensacola, FL) and GPS data logger software BT747 (www.bt747.org), respectively. All accelerometer and GPS files were processed and merged using the Personal Activity and Location Measurement System to match the 2 types of data based on their timestamp and calculate wear time and PA levels. ${ }^{18}$

To deepen our understanding of the least-active children's PA behavior during recess, we wanted to measure both MVPA, light PA (LPA), and sedentary behavior (SED). Also, a previous study using GPS devices found that children exposed to a multicomponent recess intervention only increased their time spent in LPA. ${ }^{19}$

The counts per minute (CPM) cut points defined by Evenson were used to classify PA levels for the accelerometer data: SED, 0 to 100 CPM; LPA, 101 to 2295 CPM; and MVPA, >2295 CPM. ${ }^{16}$ The Evenson cut points have previously been recommended to estimate PA intensities for children. ${ }^{20}$ Continuous periods of at least 60 minutes of 0 values were classified as accelerometer nonwear time and were removed from the analysis. ${ }^{21}$ The combined data were then downloaded into a purpose-built PostgreSQL database and combined with data from class timetables and schoolyard GIS data to categorize all data points into time spent in school, recess, and schoolyard. During the analysis, recess was defined as the sum of all breaks (including lunch) per day for each child. The 
Table 1 Descriptions of Intervention Components and Photos of the 6 Schoolyards After Renewal

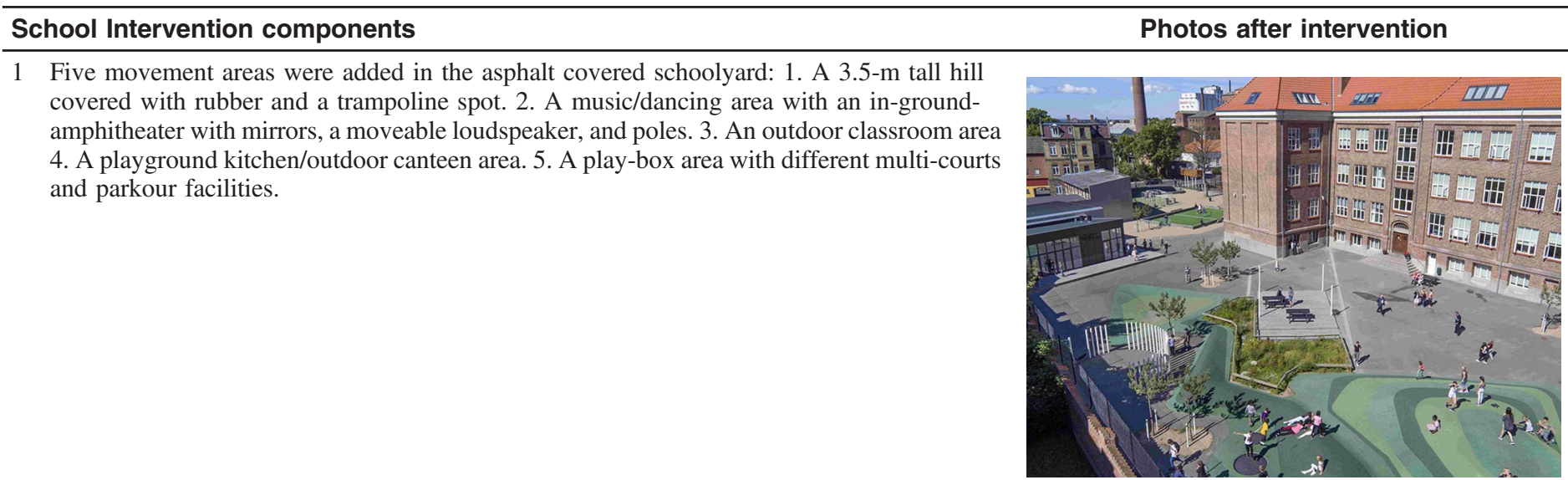

2 A loop was made to merge a forest area and the schoolyard. The loop runs through the schoolyard and the forest, and it varies in the design. It takes form as a bench, a tribune, a broken climbing-ladder, swings, a spider's web, balance-bars, and a treetop house. Along the loop, different locations are found such as a forest-café, a forest-amphitheater, a skating pool, a multi-court area, and a dancing spot with a big screen.

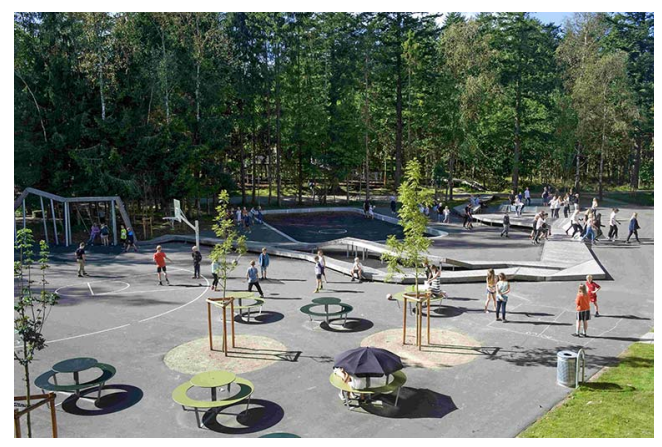

3 A slope was made to connect the schoolyard and a forest area. The slope contains learning locations with QR-codes supplemented with an App. The slope runs by several activity locations such as balance-bars, a climbing-net, swings in the trees, trampolines, and an obstacle course.

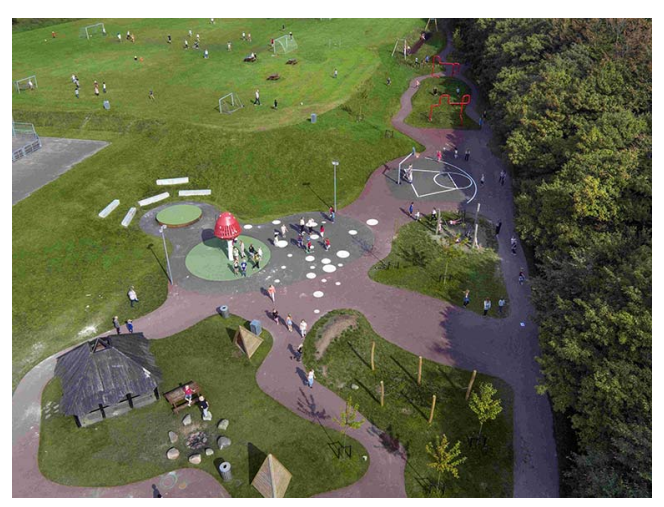

4 Three different types of landscapes were added in the schoolyard: 1 . The mountain area consists of several hills covered with rubber, a cave with a climbing wall inside, and a skate area. 2. The forest area consists of trees, a high climbing tower, and hammocks. 3. The smallcity area includes playhouses and balance-bars.

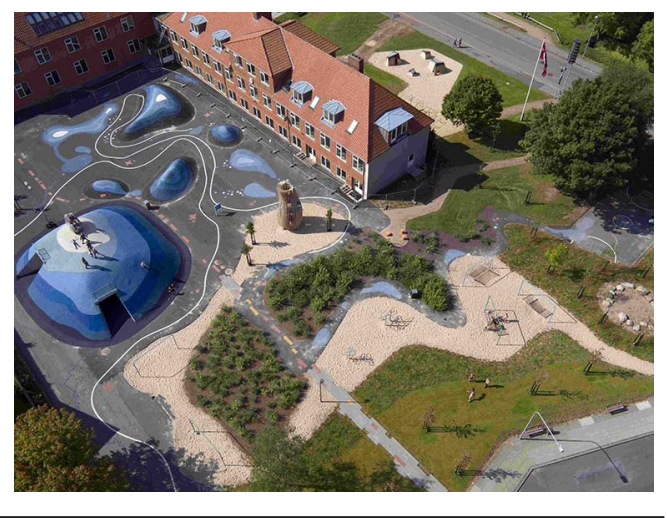


Table 1 (continued)

\section{School Intervention components}

5 A simple $166 \mathrm{~m}^{2}$ "super furniture" was built in a forest area including platforms, canopy, stairs, and a shed with basic equipment for playing and teaching.
Photos after intervention

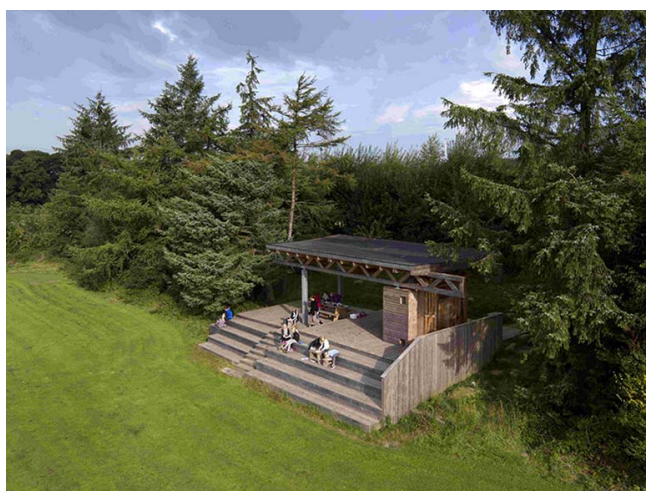

6 A multi-court was built surrounded by activity gables for climbing and hanging out. A huge gymnastic mat was placed in the multi-court area and markings were made on the surrounding asphalt surface.

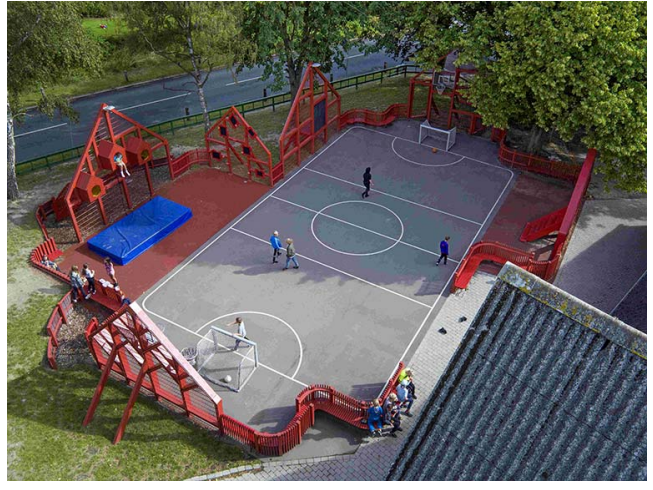

schoolyards were defined as all outdoor areas at the school grounds mapped in GIS. Days with at least $80 \%$ of combined accelerometer and GPS wear time data at school, during school hours, were considered valid and included in the analysis. ${ }^{22}$ The data processing resulted in 4 recess time variables: total recess time per day, at school and with PA measurement; MVPA during recess at the school; LPA during recess at the school; and SED during recess at the school. The same variables were calculated for time and activities in the schoolyard during recess.

Based on mean minutes of recess MVPA per child per school, the least-active children were defined as all children in the lowest activity quartile at baseline and follow-up, respectively. All analyses were performed using Stata/IC (version 15; StataCorp LLC, College Station, TX) in 2018. General linear mixed models were used to test differences in schoolyard time and PA levels during recess before and after the renewals for the least-active children. Analyses were conducted with both absolute minutes and the proportion of minutes in each PA level out of total schoolyard time, as separate independent variables. The analyses were adjusted for differences in age, gender, and number of valid days. "Minutes of schoolyard PA during recess per day" was the unit of the analysis, and days from the same person were treated as a repeated measure with a random effect. "School" was included as a fixed clustering effect, because of the limited number of schools participating, and "class-level" clustering was included as a random effect. The residual plots of each model were evaluated for normal distributions. To account for mild right skewness, 2 outcomes were $\log$ transformed to achieve a normal distribution. Significance levels were set at $P \leq .05$.

\section{Results}

Parental consent was received for 604 children (50.9\%) at baseline and $494(60.5 \%)$ at follow-up. A total of 14 children at baseline and 27 at follow-up were excluded because of device error. In addition, 37 children at baseline and 28 at follow-up were excluded, as they spent less than $80 \%$ of at least one school day at school wearing the devices. The sample with valid data consisted of 553 children (54\% girls) at baseline and 439 children (56\% girls) at follow-up. A characteristic of the whole sample of children, across the 6 schools, prior to and following schoolyard renewals, is found in Table 2.

At baseline, 135 children ( $70 \%$ girls) were categorized in the group of least-active children and 108 (76\% girls) at follow-up. At baseline, $24 \%$ of the least-active children attended grades 4 to 5 (10-12 y old), $46 \%$ attended grades 6 to 7 (12-14 y old), and 30\% attended grades 8 to 9 (14-16 y old). At follow-up, the distribution was $33 \%, 44 \%$, and $23 \%$, respectively (Table 2 ).

The least-active children generated 418 days of combined accelerometer and GPS data at baseline, with data corresponding to an average of 3.1 valid days per child with a mean of 12.2 hours daily wear time. At follow-up, 421 days with combined data were generated with an average of 3.9 valid days per child with a mean of 11.4 hours daily wear time. Children spent an average of $44.5 \mathrm{~min}$ utes daily in MVPA, corresponding to $6.0 \%$ of daily time at baseline. At follow-up, participants generated an average of 36.9 minutes in MVPA, corresponding to $5.3 \%$ of daily time. At baseline, $3.9 \%$ of the daily MVPA was generated during recess for the least-active children, and after renewals, recess MVPA amounted to $11.0 \%$ of their daily MVPA (Table 2). 
Table 2 Sample Characteristics Prior to and Following Schoolyard Renewals

\begin{tabular}{|c|c|c|}
\hline Sample characteristics & $\begin{array}{c}2014 \\
\text { Mean (SD/\%) }\end{array}$ & $\begin{array}{c}2016 \\
\text { Mean (SD/\%) }\end{array}$ \\
\hline Schools participating & 6 & 6 \\
\hline Participants invited & 1186 & 816 \\
\hline Parental consent & 604 & 494 \\
\hline Participants with GPS and accelerometer data & 590 & 467 \\
\hline Participants with valid days (\% girls) & $553(54 \%)$ & $439(56 \%)$ \\
\hline Least-active children ( $\%$ girls $)$ & $135(70 \%)$ & $108(76 \%)$ \\
\hline Grade $4-5 / 10-12$ y old (\% proportion) & $33(24 \%)$ & $36(33 \%)$ \\
\hline Grade $6-7 / 12-14$ y old (\% proportion) & $57(46 \%)$ & $48(44 \%)$ \\
\hline Grade $8-9 / 14-16$ y old (\% proportion) & $45(30 \%)$ & $24(23 \%)$ \\
\hline Sum valid days & 418 & 421 \\
\hline Mean valid days $(\mathrm{CI})$ & $3.1(2.3-3.3)$ & $3.9(3.6-4.2)$ \\
\hline Mean wear time daily in minutes, min & $730.9(159.8)$ & $685.7(154.3)$ \\
\hline Mean daily MVPA, min & $44.5(24.1)$ & $36.9(21.3)$ \\
\hline Mean daily LPA, min & $179.9(63.0)$ & $160.6(63.5)$ \\
\hline Mean daily SED, min & $506.5(114.3)$ & $488.2(107.4)$ \\
\hline Proportion MVPA/day wear, $\%$ & $6.0 \%$ & $5.3 \%$ \\
\hline Mean recess wear time daily, min & $44.2(14.7)$ & $64.4(11.1)$ \\
\hline Mean daily recess MVPA, min & $1.6(1.6)$ & $3.5(2.2)$ \\
\hline Mean daily recess LPA, min & $11.8(8.1)$ & $18.7(8.8)$ \\
\hline Mean daily recess SED, min & $30.8(10.9)$ & $42.2(15.0)$ \\
\hline Proportion recess MVPA/daily MVPA, \% & $3.9 \%$ & $11.0 \%$ \\
\hline
\end{tabular}

Abbreviations: 2014, Baseline (prior renewals); 2016, Follow-up (after renewals); CI, confidence interval; LPA, light physical activity; MVPA, moderate to vigorous physical activity; SED, sedentary behavior. Note: Mean minutes wear time = mean minutes of combined accelerometer and GPS data per day per child at valid days; Participants invited $=$ children representing the target group for the schoolyard renewals at each school at the given time; valid days $=$ days with at least $80 \%$ of combined accelerometer and GPS data at school during school hours.

The least-active children spent $37.8 \%$ of recess time in their schoolyard postrenewal, a difference of $6.7 \%$ compared with baseline $(P=.05)$. The absolute difference in schoolyard time following renewals corresponded to 12.1 minutes more daily $(P<.05)$, on average. The least-active children also accumulated 4.4 minutes more PA daily after renewals $(P<.05)$, on average (a difference of $0.9 \mathrm{~min}$ in MVPA and $3.5 \mathrm{~min}$ in LPA). With a schoolyard time of 24.5 minutes on average per day during recess after renewals, the least-active children accumulated the same amount of time in the schoolyard as all children on average (25.7 min, not shown in the table). However, the proportion of time spent in each PA level in the schoolyard during recess differed. After renewals, the least-active children spent $7.0 \%$ of their schoolyard time during recess in MVPA, a difference of $2.0 \%$ compared with baseline $(P<.05)$ and $60.3 \%$ of time was spent being sedentary, a difference of $5.5 \%(P<.05)$ (Table 3$)$. In comparison, the proportion of MVPA and SED for all children during recess was $16.6 \%$ and $37.5 \%$ of the schoolyard time, respectively (not shown in the table).

\section{Discussion}

The aim of this study was to explore how schoolyard renewals influenced schoolyard use and PA among the least-active children during recess. Compared with the least-active children at baseline, the least-active children following renewals accumulated more time and PA in the schoolyard during recess, both absolute and proportional. However, the positive difference in schoolyard PA found for the least-active children was relatively small compared with the difference in schoolyard PA for all children. In the following sections, the findings and the potential implications for practice and future research are discussed.

\section{The Least-active Children}

In this study, we found that the least-active children were a mixed group of children from different grades and genders. Similar to this, Shervey and DiPerna ${ }^{23}$ found that the children who took the least number of steps during recess varied in both gender and age/ grade level.

At both time points in our study, the group of least-active children consisted of children from different grade levels, but with slightly more children from grades 6 to 7 , being 12-14 years old. In line with this, a review by Ridgers et $\mathrm{al}^{24}$ found no association between grade or age and recess PA. In contrast, a study found a difference in recess PA among grades 4 to 10 children, with grade 6 children being the most active during recess, following a decline for girls in grade 7 and boys in grade $8 .{ }^{25}$ As an explanation for age differences, a qualitative study concluded that children in grade 7 often felt more lazy and afraid of sweating compared with children in grade 5, and opted to stay sedentary in the classroom during recess for these reasons. ${ }^{26}$ In relation to recess interventions, Erwin et $\mathrm{al}^{9}$ found that PA outcomes were moderated by age with highest effect among the youngest children. These authors concluded that 
Table 3 Differences in Schoolyard PA During Recess From Baseline to Follow-up for the Least Active Children

\begin{tabular}{|c|c|c|c|c|c|c|}
\hline Outcome variables & $\begin{array}{c}2014 \\
n=135\end{array}$ & SD & $\begin{array}{c}2016 \\
n=108\end{array}$ & SD & $\begin{array}{c}\text { Adjusted differences } \\
(95 \% \mathrm{Cl})\end{array}$ & $P$ value \\
\hline \multicolumn{7}{|l|}{$\begin{array}{l}\text { Minutes of total schoolyard wear } \\
\text { time and in PA levels per day }\end{array}$} \\
\hline Wear time in schoolyard, min & 14.8 & 13.5 & 24.5 & 16.3 & $12.1(9.4$ to 14.8$)$ & $<.001$ \\
\hline MVPA, min & 0.8 & 1.5 & 1.6 & 2.1 & $0.9(0.6$ to 1.3$)$ & $<.001$ \\
\hline LPA, min & 4.4 & 5.7 & 7.6 & 7.0 & $3.5(2.4$ to 4.7$)$ & $<.001$ \\
\hline $\mathrm{SED}, \min$ & 9.6 & 9.6 & 15.3 & 12.8 & 7.7 (5.6 to 9.7$)$ & $<.001$ \\
\hline \multicolumn{7}{|c|}{$\begin{array}{l}\text { Proportion of recess time in PA levels } \\
\text { for time spent in the schoolyard }\end{array}$} \\
\hline Wear time in schoolyard, $\%$ & 33.0 & 27.35 & 37.8 & 23.9 & $6.7(1.8$ to 11.5$)$ & .007 \\
\hline MVPA, $\%$ & 4.6 & 8.0 & 7.0 & 8.6 & $2.0(0.8$ to 3.3$)$ & .002 \\
\hline LPA, \% & 27.6 & 20.21 & 32.7 & 20.3 & $3.4(-0.1$ to 6.9$)$ & .054 \\
\hline SED, \% & 67.7 & 24.6 & 60.3 & 24.4 & $-5.5(-9.5$ to 1.6$)$ & .006 \\
\hline
\end{tabular}

Abbreviations: 2014, Baseline (prior renewals); 2016, Follow-up (after renewals); CI, confidence interval; LPA, light PA; MVPA, moderate to vigorous PA; PA, physical activity; SED, sedentary behavior. Note: Wear time in the schoolyard = the sum of wear time in the schoolyard during all breaks per day. Multilevel models adjusted for age, gender, and number of valid days. School and class level were included in the model to account for clustering; recess schoolyard time and PA per day per child were included as repeated measurements.

the initiatives employed in the interventions possibly appealed more to the younger children.

At both time points, girls formed the majority of the leastactive group of children representing $70 \%$ at baseline and $76 \%$ at follow-up. In line with this, multiple studies have found girls to be less active than boys during recess. ${ }^{7,24,27,28}$ In previous studies, boys have also been observed using the schoolyard more actively than girls after schoolyard modifications..$^{29,30}$ Studies have reported that boys dominate schoolyards during recess, which limits the opportunities for many girls to actively use the schoolyard features. ${ }^{31-34}$ In a previous study, we also found several barriers to recess PA solely expressed by girls such as lack of adult-organized recess activities and lack of facilities for social activities in the schoolyard. ${ }^{35}$ It is important to be aware, however, that the group of least-active children also included boys. In a previous study, we observed a hierarchy among boys where not being skilled and sporty implied a lower status in the schoolyard. That made some boys feel rejected from schoolyard play, and they preferred to stay in the classroom during recess playing computer games. ${ }^{31}$

\section{Schoolyard Time and PA}

The least-active children at follow-up spent more time in the schoolyard (6.7\%) compared with the least-active children at baseline, and they spent $37.8 \%$ of their recess time in the schoolyard after renewal. The positive difference in recess time in the schoolyard might also explain the positive difference in recess PA in the schoolyard following renewals. A review found time spent outdoors to be associated with higher daily PA levels, ${ }^{36}$ as well as during recess. ${ }^{27}$

It seems plausible that the improved schoolyards with new and more playground equipment influenced the least-active children's recess behavior. This finding is in line with a study that showed an association between the overall number of features in the schoolyard and recess PA. ${ }^{37}$ Also, a review concluded that the largest change in children's PA levels before and after intervention occurred by the addition of playground equipment. ${ }^{9}$ However, neither of the studies divided the children into subgroups based on PA levels, thus comparing their results with the least-active children in our study can be challenging. In a case study, however, we conducted before the renewals; we found that the low activity children mostly stayed indoors during recess because they felt a lack of outdoor play facilities. ${ }^{38}$ This indicates that adding play facilities in the schoolyard had an impact on outdoor time and PA during recess among the least-active children.

As part of evaluating the Activating Schoolyards Study, we investigated specific areas and features with high levels of PA in 3 of the renewed schoolyards using accelerometer and GPS. Across schools, we found that ballgame courts, foursquare markings, and hills generated high activity for both genders. Girls were also active using dancing facilities, multi-courts, nature-inspired elements, and a spiderweb, whereas boys also actively played in-between facilities and on obstacle trails. ${ }^{39}$ This case-based analysis was not accomplished explicitly on the group of the least-active children to avoid very small samples sizes. To design schoolyards promoting low-activity children's recess PA, the next step in research should be to investigate what kind of play facilities motivates these children.

In spite of the positive difference in the least-active children's PA levels in the schoolyard after renewals, the difference was relatively small compared with the difference in PA levels for all participating children. The small difference in schoolyard PA found in the present study could indicate that PA does not happen spontaneously for the least-active children. Therefore, solely improving the physical environment might have limited impact on the least-active children's recess PA. Seen from an ecological perspective, it is important to handle the complex interrelations between the least-active children and the entire schoolyard environment. ${ }^{40}$ In particular, it seems as if the social environment has an impact on recess PA among the group of least-active children. Previous studies have found a hierarchy in the schoolyard where the least-skilled and sporty boys and girls felt excluded from recess play. ${ }^{31-33}$ Focusing more on integrating active schoolyard designs with social support, such as adult-facilitated games, may yield greater impact on PA for the least-active children. ${ }^{35}$ Other studies also suggest that the physical schoolyard environment should be supported by social activities to increase recess PA. ${ }^{41,42}$ Stellino et $\mathrm{al}^{43}$ suggest schools to consider demographic factors in the 
creation of recess opportunities to increase PA. Thus, it is important to note that a one-size-fits-all approach should be avoided. ${ }^{9}$

In summary, even when PA increases for all children, different mechanisms seem to apply for different groups. If we aim to promote recess PA for the least-active children, we might need to lower our expectations of what the changes to the physical environment can do alone, and focus more on investigating the interplay of various mechanisms such as design, features, recess rules, social support, social interaction, and interests.

\section{Strengths and Limitations}

As far as we know, this study is the first schoolyard intervention study focusing specifically on the recess PA behavior of the leastactive children before and after intervention. Furthermore, it is one of the first intervention studies in this field using a combination of accelerometer and GPS. ${ }^{19,39}$ In the current study, we used the combination of accelerometer, GPS, and GIS to determine the level of PA and the proportion of recess time spent outdoors in the schoolyard. It increased the validity of our findings, since most Danish children in the target group can choose whether they want to stay indoors or outdoors during recess.

It is important to notice that only about half of all children attending the 6 schools participated in the baseline study (50.9\%). Following challenges with recruitment at baseline, additional efforts were made at follow-up (ie, study information to all teachers on a teacher meeting and a prize to the class with most participants), resulting in a larger participation rate after renewals (60.5\%).

Moreover, the study used a cross-sectional design focusing on the same age group at 2 time points to avoid the age-dependent decline in PA. ${ }^{13}$ This means that we compared 2 different samples of children that made selection bias a possibility. Furthermore, no control groups were included in the study.

Other influencing factors might also have had an impact on the objectively assessed PA, for example, rain during measurement days or changed recess policies in the schools. Furthermore, the intervention was developed using a participatory approach and was tailored to the needs of the particular school. Using a participatory design to develop tailored interventions has proven to be an effective and viable approach, ${ }^{44}$ but it also makes the results more difficult to interpret. Thus, the results should be interpreted cautiously, and direct causality cannot be inferred.

We selected the least-active children based on their recess PA and, potentially, these children could only have been the least active in that domain. A strength, however, is that we tested that the least-active children during recess were also among the least-active children when looking at overall PA levels.

Finally, using accelerometers monitoring intensity of PA, our data suggest that the cardiorespiratory system increased, while we do not know if mechanical stress was influenced. Future recess studies should implement direct observation to discern what activities the least-active children engage in. This would help further clarify the complexity of PA.

\section{Conclusions}

It has been unclear if recess interventions promoting PA were equally effective for all children, or if they "just" provided more opportunities for the children who were already active. Recess time and PA in the schoolyard were more accumulated for the least-active children, as well as for all children, after renewals compared with baseline. Adding various play facilities in the schoolyard seems to have an impact on outdoor time and PA during recess among the least-active children. However, the difference in schoolyard PA found for the least-active children was relatively small compared with the difference for all children. This could indicate that physical improvements in the schoolyard had limited impact on the leastactive children's recess PA. Therefore, a one-size-fits-all approach should be avoided. To increase the equity of this type of interventions and achieve better results for the children most in need, future studies should investigate the complex interrelations between the least-active children and the entire schoolyard environment.

\section{Acknowledgments}

The authors thank all the participating children and their parents for taking part in the study. The authors also thank the school staff for their assistance in organizing the data collection. This work was supported by the Danish foundation Realdania and the Danish foundation for Culture and Sport Facilities.

\section{References}

1. Strong WB, Malina RM, Blimkie CJR, et al. Evidence based physical activity for school-age youth. J Pediatr. 2005;146(6):732-737. PubMed ID: 15973308 doi:10.1016/j.jpeds.2005.01.055

2. Aubert S, Barnes JD, Abdeta C, et al. Global matrix 3.0 physical activity report card grades for children and youth: results and analysis from 49 countries. J Phys Act Health. 2018;15(suppl 2):S251-S273. PubMed ID: 30475137 doi:10.1123/jpah.2018-0472

3. Telama R, Yang X, Viikari J, Valimaki I, Wanne O, Raitakari O. Physical activity from childhood to adulthood: a 21-year tracking study. Am J Prev Med. 2005;28(3):267-273. PubMed ID: 15766614 doi:10.1016/j.amepre.2004.12.003

4. Ridgers ND, Stratton G, Fairclough SJ. Physical activity levels of children during school playtime. Sports Med. 2006;36(4):359-371. PubMed ID: 16573359 doi:10.2165/00007256-200636040-00005

5. Dobbins M, Husson H, DeCorby K, LaRocca RL. School-based physical activity programs for promoting physical activity and fitness in children and adolescents aged 6 to 18. Cochrane Database Syst Rev. 2013;(2):CD007651. PubMed ID: 23450577 doi:10.1002/ 14651858.CD007651.pub2

6. Erwin H, Abel M, Beighle A, Noland MP, Worley B, Riggs R. The contribution of recess to children's school-day physical activity. $J$ Phys Act Health. 2012;9(3):442-448. PubMed ID: 21934153 doi:10. 1123/jpah.9.3.442

7. Fairclough SJ, Beighle A, Erwin H, Ridgers ND. School day segmented physical activity patterns of high and low active children. BMC Public Health. 2012;12(1):406. PubMed ID: 22672654 doi:10. 1186/1471-2458-12-406

8. Cox M, Schofield G, Greasley N, Kolt GS. Pedometer steps in primary school-aged children: a compar of school-based and outof-school activity. J Sci Med Sport. 2006;9(1-2):91-97. PubMed ID: 16580252 doi:10.1016/j.jsams.2005.11.003

9. Erwin HE, Ickes M, Ahn S, Fedewa A. Impact of recess interventions on children's physical activity-a meta-analysis. Am J Health Prom. 2014;28(3):159-167. PubMed ID: 23875990 doi:10.4278/ajhp. 120926-LIT-470

10. Ridgers ND, Stratton G, Fairclough SJ, Twisk JWR. Long-term effects of a playground markings and physical structures on children's recess physical activity levels. Prev Med. 2007;44(5):393-397. PubMed ID: 17335891 doi:10.1016/j.ypmed.2007.01.009 
11. Ridgers ND, Stratton G, Fairclough SJ, Twisk JWR. Children's physical activity levels during school recess: a quasi-experimental intervention study. Int J Behav Nutr Phys Act. 2007;4(1):19. PubMed ID: 17517136 doi:10.1186/1479-5868-4-19

12. Andersen HB, Pawlowski CS, Scheller HB, Troelsen J, Toftager M, Schipperijn J. Activating schoolyards: study design of a quasiexperimental schoolyard intervention study. BMC Public Health. 2015; 15(1):523. PubMed ID: 26026650 doi:10.1186/s12889-015-1828-9

13. Farooq MA, Parkinson KN, Adamson AJ, et al. Timing of the decline in physical activity in childhood and adolescence: Gateshead Millennium Cohort Study. Br J Sports Med. 2017; 52(15):1002-1006. PubMed ID: 28288966 doi:10.1136/bjsports2016-096933

14. Pawlowski CS, Andersen HB, Arvidsen J, Schipperijn J. Changing recess geographies: children's perceptions of schoolyard renovation project promoting physical activity. Children's Geographies. 2019;17(6):664-675. doi:10.1080/14733285.2019.1582754

15. McCrorie PRW, Fenton C, Ellaway A. Combining GPS, GIS, and accelerometry to explore the physical activity and environment relationship in children and young people-a review. Int J Behav Nutr Phys Act. 2014;11(1):93. PubMed ID: 25356782 doi:10.1186/ s12966-014-0093-0

16. Evenson KR, Catellier DJ, Gill K, Ondrak KS, McMurray RG. Calibration of two objective measures of physical activity for children. J Sports Sci. 2008;26(14):1557-1565. PubMed ID: 18949660 doi:10.1080/02640410802334196

17. Schipperijn J, Kerr J, Duncan S, Madsen T, Klinker CD, Troelsen J. Dynamic accuracy of GPS receivers for use in health research: a novel method to assess GPS accuracy in real-world settings. Front Public Health. 2014;2:21. PubMed ID: 24653984 doi:10.3389/fpubh.2014. 00021

18. Carlson JA, Jankowska MM, Meseck K, et al. Validity of PALMS GPS scoring of active and passive travel compared with SenseCam. Med Sci Sports Exerc. 2015;47(3):662-667. PubMed ID: 25010407 doi:10.1249/MSS.0000000000000446

19. Van Kann DHH, de Vries SI, Schipperijn J, de Vries NK, Jansen MWJ, Kremers SPJ. A multicomponent schoolyard intervention targeting children's recess physical activity and sedentary behavior: effects after 1 year. J Phys Act Health. 2017;14(11):866-875. PubMed ID: 28682695 doi:10.1123/jpah.2016-0656

20. Trost SG, Loprinzi PD, Moore R, Pfeiffer KA. Comparison of accelerometer cut points for predicting activity intensity in youth. Med Sci Sports Exerc. 2011;43(7):1360-1368. PubMed ID: 21131873 doi:10.1249/MSS.0b013e318206476e

21. Adams MA, Johnson WD, Tudor-Locke C. Steps/day translation of the moderate-to-vigorous physical activity guideline for children and adolescents. Int J Behav Nutr Phys Act. 2013;10(1):49. PubMed ID: 23601369 doi:10.1186/1479-5868-10-49

22. Catellier DJ, Hannan PJ, Murray DM, et al. Imputation of missing data when measuring physical activity by accelerometry. Med Sci Sports Exerc. 2005;37(11 Suppl):S555-S562. PubMed ID: 16294118 doi:10.1249/01.mss.0000185651.59486.4e

23. Shervey SW, DiPerna JC. Engagement in physical activity during recess: gender and grade level differences in the elementary grades. $J$ Phys Act Health. 2017;14(9):677-683. PubMed ID: 28513317 doi:10.1123/jpah.2014-0499

24. Ridgers ND, Salmon J, Parrish AM, Stanley RM, Okely AD. Physical activity during school recess: a systematic review. Am J Prev. 2012;43(3):320-328. PubMed ID: 22898126 doi:10.1016/j.amepre. 2012.05.019

25. Haug E, Torsheim T, Sallis JF, Samdal O. The characteristics of the outdoor school environment associated with physical activity. Health
Educ Res. 2010;25(2):248-256. PubMed ID: 18936270 doi:10.1093/ her/cyn050

26. Ladekjaer E. Børn i bevagelse. Odense, Denmark: Department of Sports Science and Clinical Biomechanics, University of Southern Denmark; 2016.

27. Dessing D, Pierik FH, Sterkenburg RP, van Dommelen P, Maas J, de Vries SI. Schoolyard physical activity of 6-11 year old children assessed by GPS and accelerometry. Int J Behav Nutr Phys Act. 2013;10(1):97. PubMed ID: 23945145 doi:10.1186/1479-5868-10-97

28. Nielsen G, Pfister G, Andersen LB. Gender differences in the daily physical activities of Danish school children. Eur Phys Educ Rev. 2011;17(1):69-90. doi:10.1177/1356336X11402267

29. Colabianchi N, Kinsella AE, Coulton CJ, Moore SM. Utilization and physical activity levels at renovated and unrenovated school playgrounds. Prev Med. 2009;48(2):140-143.PubMed ID: 19063915 doi:10.1016/j.ypmed.2008.11.005

30. Anthamatten P, Brink L, Lampe S, Greenwood E, Kingston B, Nigg C. An assessment of schoolyard renovation strategies to encourage children's physical activity. Int J Behav Nutr Phy Act. 2011;8(1):27. PubMed ID: 21477325 doi:10.1186/1479-5868-8-27

31. Pawlowski CS, Ergler C, Tjornhoj-Thomsen T, Schipperijn J, Troelsen J. 'Like a soccer camp for boys': a qualitative exploration of gendered activity patterns in children's self-organized play during school recess. Eur Phys Educ Rev. 2015;21(3):275-291. doi:10.1177/ $1356336 \mathrm{X} 14561533$

32. Lucas AJ, Dyment JE. Where do children choose to play on the school ground? The influence of green design. Educ 3-13. 2010; 38(2):177-189.

33. Paechter C, Clark S. Learning gender in primary school playgrounds: findings from the Tomboy Identities Study. Pedagog Cult Soc. 2007;15(3):317-331. doi:10.1080/14681360701602224

34. Knowles ZR, Parnell D, Stratton G, Ridgers ND. Learning from the experts: exploring playground experience and activities using a write and draw technique. J Phys Act Health. 2013;10(3):406-415. PubMed ID: 22820462 doi:10.1123/jpah.10.3.406

35. Pawlowski CS, Tjornhoj-Thomsen T, Schipperijn J, Troelsen J. Barriers for recess physical activity: a gender specific qualitative focus group exploration. BMC Public Health. 2014;14(1):639. PubMed ID: 24958158 doi:10.1186/1471-2458-14-639

36. Sallis JF, Prochaska JJ, Taylor WC. A review of correlates of physical activity of children and adolescents. Med Sci Sports Exerc. 2000;32(5): 963-975. PubMed ID: 10795788 doi:10.1097/00005768-20000500000014

37. Nielsen G, Bugge A, Hermansen B, Svensson J, Andersen LB. School playground facilities as a determinant of children's daily activity: a cross-sectional study of Danish primary school children. J Phys Act Health. 2012;9(1):104-114. PubMed ID: 22232496 doi:10.1123/jpah.9.1.104

38. Pawlowski CS, Andersen HB, Troelsen J, Schipperijn J. Children's physical activity behavior during school recess: a pilot study using GPS, accelerometer, participant observation, and go-along interview. PLoS One. 2016;11(2):e0148786. PubMed ID: 26859288 doi:10. 1371/journal.pone.0148786

39. Andersen HB, Christiansen LB, Pawlowski CS, Schipperijn J. What we build makes a difference-Mapping activating schoolyard features after renewal using GIS, GPS and accelerometers. Landscape Urban Plan. 2019;191:103617.

40. McLaren L, Hawe P. Ecological perspectives in health research. J Epi Comm Health. 2005;59(1):6-14. PubMed ID: 15598720 doi:10. 1136/jech.2003.018044

41. Brittin J, Frerichs L, Sirard JR, et al. Impacts of active school design on school-time sedentary behavior and physical activity: a pilot 
natural experiment. PLoS One. 2017;12(12):e0189236. PubMed ID: 29216300 doi:10.1371/journal.pone.0189236

42. Powell E, Woodfield LA, Nevill AAM. Children's physical activity levels during primary school break times: a quantitative and qualitative research design. Eur Phys Educ Rev. 2016;22(1):82-98. doi:10. 1177/1356336X15591135
43. Stellino MB, Sinclair CD, Partridge JA, King KM. Differences in children's recess PA: recess activity of the week intervention. J School Health. 2010;80(9):436-444. PubMed ID: 20690975 doi: 10.1111/j.1746-1561.2010.00525.x

44. Minkler M, Wallerstein N. Community-Based Participatory Research for Health. San Francisco: Jossey-Bass; 2008. 